\title{
Actualización en el estudio de Granulomatosis con poliangeitis (Granulomatosis de Wegener)
}

David Ladrón de Guevaral, , Felipe Cerda², María Ángela Carreñoº , Antonio Piottante ${ }^{4}$, Patricia Bitar'.

1. Departamento de Radiología, Clínica Las Condes, Santiago, Chile.

2. Becado de Radiología, Universidad de Chile, Santiago, Chile.

3. Departamento de Medicina Interna, Unidad de Reumatología, Clínica Las Condes, Santiago, Chile.

4. Departamento de Anatomía Patológica, Clínica Las Condes, Santiago, Chile.

\section{Update in study of Granulomatosis with polyangiitis (Wegener's granulomatosis)}

Resumen: La granulomatosis con poliangeítis (GPA) es una vasculitis sistémica de pequeño vaso, que afecta más frecuentemente el tracto respiratorio y el riñón. Sus criterios diagnósticos se basan en la clínica, exámenes de laboratorio, imágenes e histología. El 90\% son ANCA (anticuerpos anticitoplasma de neutrófilos) positivos. La histología muestra inflamación granulomatosa, necrosis y vasculitis. Los exámenes de imagen son de vital importancia en su estudio inicial y seguimiento, correspondiendo principalmente a técnicas tomográficas. La tomografía Computada (TC) es el método de elección para la evaluación de vía aérea superior y pulmón, con alta sensibilidad en afectación de cavidades nasal/paranasales, árbol bronquial y pulmón. La Resonancia Magnética está indicada en compromiso del sistema nervioso central y corazón. EI PET/CT presenta alta sensibilidad en enfermedad tóraco-abdominal, es de utilidad en detectar lesiones no visibles con otras técnicas, y en control de tratamiento. El compromiso renal, de alta ocurrencia en GPA, presenta escasa traducción en las imágenes y es frecuentemente indetectable con imágenes, aunque el PET/CT puede ser positivo en casos de glomerulonefritis acentuada. La radiología simple no debe ser utilizada en el estudio de GPA dado su bajo rendimiento diagnóstico. El tratamiento se basa en terapia corticoidea e inmunosupresora. Las recaídas son frecuentes, por lo que estos pacientes requieren seguimiento a largo plazo.

Palabras clave: Granulomatosis con poliangeítis, Granulomatosis de Wegener, vasculitis, Positron emission tomography, Tomografía computada.

\footnotetext{
Abstract: Granulomatosis with polyangiitis (GPA) is a systemic type of vasculitis that affects small vessels, most commonly involving the respiratory tract and kidneys. Diagnosis is based on clinical criteria, laboratory tests, imaging and histology. Ninety percent are ANCA (anti-neutrophilic cytoplasmic antibodies) positive. Histology demonstrates granulomatous inflammation, necrosis and vasculitis. Imaging studies are vital for the initial work-up and follow-up. Computed Tomography (CT) is the method of choice for evaluation of the upper airway and lungs, because of its high sensitivity detecting anomalies of paranasal sinuses, bronchial tree and lungs. Magnetic Resonance is indicated for evaluation of the central nervous system and heart. PET/CT has high sensitivity for thoracic and abdominal disease, is useful at detecting lesions not seen with other imaging techniques, and for follow-up. Renal involvement, very frequent on GPA, is usually undetectable at imaging, but may be seen at PET/CT in cases of marked glomerulonephritis. Plain $X$-rays should not be used for evaluation of GPA because of their low diagnostic performance. Treatment is based on corticosteroid and immunosuppressive therapy. Relapses are frequent, so these patients require long-term follow-up.

Key words: Computed tomography, Granulomatosis with polyangiitis, Positron emission tomography, Wegener's granulomatosis, vasculitis.

Ladrón de Guevara $D$, et al. Actualización en el estudio de Granulomatosis con poliangeitis (Granulomatosis de Wegener). Rev Chil Radiol 2019; 25(1): 26-34.

${ }^{*}$ Correo electrónico: David Ladrón de Guevara H./ dlg@clc.cl.

Trabajo enviado el 09 de enero de 2019. Aceptado para publicación el 23 de marzo de 2019.
} 


\section{Introducción}

Las vasculitis son un grupo de enfermedades caracterizadas por inflamación de las paredes de los vasos sanguíneos. Según el consenso de Chapel Hill de 2012 que las clasifica según el tipo de vaso que predominantemente afecta, las vasculitis de pequeño vaso comprometen pequeñas arterias intraparenquimatosas, arteriolas, capilares y vénulas pudiendo afectar también vasos de mediano calibre. Dentro de este grupo se encuentran las vasculitis asociadas a anticuerpos anticitoplasma de neutrófilos (ANCA), cuyos mayores exponentes son la poliangeítis microscópica, granulomatosis con poliangeítis (GPA) (ex Wegener), granulomatosis con poliangeítis eosinofílica (Churg Strauss) y las limitadas a un órgano específico ${ }^{(1)}$.

La GPA es una vasculitis granulomatosa necrotizante sistémica asociada a ANCA cuyas lesiones afectan principalmente el tracto respiratorio y el riñón ${ }^{(2)}$. Puede presentarse a cualquier edad con un peak a los 65-74 años. Su incidencia anual es de 5-10/millón con una prevalencia de 24-157 casos por millón, siendo similar entre ambos sexos ${ }^{(3,4)}$. En la etiopatogenia estarían involucrados gatillantes infecciosos, ambientales o farmacológicos que, en personas genéticamente predispuestas, generan una respuesta inflamatoria y producción de ANCA contra proteinasa-3 (PR3) y mieloperoxidasa (MPO) en el $80 \%$ y $10 \%$ de los pacientes respectivamente, desarrollando inflamación granulomatosa necrotizante ${ }^{(4)}$.

\section{Manifestaciones clínicas}

La enfermedad se puede presentar con síntomas constitucionales inespecíficos: malestar general, mialgias, artralgias, anorexia, baja de peso. Afecta distintos órganos, siendo lo más frecuente el compromiso de vía aérea y renal. La afectación de la vía aérea alta es lo más común y característico $(70-100 \%$ al momento del diagnóstico), principalmente a nivel nasal y sinusal, pudiendo manifestarse con descarga nasal, epistaxis, úlceras nasales, perforación del septum nasal, lesiones granulomatosas o destrucción del cartílago facial con deformación del puente nasal, inflamación sinusal o parasinusal. También puede presentar otitis media crónica, estenosis glótica o subglótica. En la vía aérea baja puede manifestarse con tos, disnea, obstrucción bronquial, y a nivel pulmonar, presente en $50-90 \%$ de los pacientes, se pueden encontrar nódulos, cavitaciones, infiltrados, pleuritis o derrame pleural y hemorragia por capilaritis alveolar. Un $40-100 \%$ de los pacientes puede tener afectación renal, caracterizada por glomerulonefritis necrotizante segmentaria con formación de crecéntica pauci-inmune (ausencia de depósitos de inmunoglobulina o complemento en la inmunofluorescencia) manifestándose con hematuria, proteinuria y falla renal. También puede haber compromiso cutáneo (vasculitis leucocitoclástica, púrpura, infartos, úlceras, nódulos), mucocutáneo (ulceras, granulomas orales), musculoesquelético (mialgias, artralgias, artritis), ocular (escleritis, epiescleritis, uveítis, alteraciones retinales, trombosis retinal, masas granulomatosas orbitales, ceguera) y urogenital (prostatitis, orquitis, epididimitis, pseudotumores, estenosis, ulceraciones). A nivel de sistema nervioso, puede tener manifestaciones centrales y periféricas, incluyendo accidente cerebrovascular, masas cerebrales, convulsiones, meningitis, parálisis de nervios craneales, neuropatía sensitivo o motora, mononeuritis múltiple. Menos frecuentes son las manifestaciones cardiovasculares (pericarditis, cardiomiopatía, cardiopatía isquémica o valvular) y gastrointestinales (vasculitis mesentérica, ulceras, perforaciones) ${ }^{(2,4)}$. En general, se pueden distinguir dos tipos de presentación de GPA: localizada o limitada, con compromiso limitado a la vía aérea alta, y la forma sistémica o difusa, con compromiso renal, pulmonar y síntomas constitucionales ${ }^{(2)}$.

\section{Criterios diagnósticos}

Para el diagnóstico es necesario considerar las manifestaciones clínicas que sugieren presencia de vasculitis, determinación de ANCA y evidencia histopatológica del órgano comprometido ${ }^{(4)}$. En el año 1990, la ACR (American College of Rheumatology) definió 4 criterios, de los cuales debe haber al menos 2 para definir GPA: 1) compromiso sinusal, 2) alteraciones en la radiología pulmonar, 3) alteración del sedimento urinario (hematuria, cilindros hemáticos), 4) histología con presencia de granulomas perivasculares, con una sensibilidad y especificidad de $88 \%$ y $92 \%{ }^{(2)}$.

Si bien la determinación de ANCA apoya el diagnóstico de GPA, en el $10 \%$ de los pacientes no es detectable. La determinación de las variaciones en los títulos de ANCA en la evolución de la enfermedad no ha demostrado ser útil para predecir recaídas, pero la presencia de títulos persistentemente elevados podría ser predictor ${ }^{(2)}$. La diferenciación entre MPO-ANCA y PR3-ANCA podría predecir la respuesta a distintos tipos de inmunosupresores ${ }^{(5)}$.

\section{Histología}

Las manifestaciones histológicas de la GPA son variadas, pero en términos generales, está conformada por tres elementos. Inflamación, necrosis y vasculitis $^{(6,7)}$.

La inflamación se caracteriza por ser un proceso que incluye elementos de inflamación crónica como linfocitos, células plasmáticas e histiocitos, algunos de los cuales conforman una reacción granulomatosa pobremente definida asociada a células gigantes multinucleadas (Figura 1). Se acompaña de inflamación aguda constituída por neutrófilos, microabscesos y numerosos eosinófilos. En la periferia la lesión se suele identificar hemorragia antigua y focos de bronquiolitis obliterante- neumonía organizada. 
La necrosis suele ser de tipo geográfico y de fondo sucio (detritus celulares) otorgándole un aspecto basofílico. A diferencia de otros procesos, dicha necrosis no tiene necesariamente relación con la reacción granulomatosa.

Los fenómenos vasculíticos suelen predominar en arteriolas y vénulas y pueden contener los mismos elementos inflamatorios previamente descritos (Figura 1). Su presencia no es específica, ni tampoco es indispensable para hacer el diagnóstico.

\section{Rol de las imágenes}

La GPA es una vasculitis que puede afectar múltiples órganos y sistemas, sin embargo, muestra una clara predilección por el sistema respiratorio, en $92 \%$ de los pacientes, y los riñones, en $80 \%$ de los casos ${ }^{(8)}$. Para su estudio imagenológico se emplean principalmente técnicas tomográficas: Tomografía Computada (TC), PET/CT (Positron Emission Tomography/Computed tomography), y Resonancia Magnética (RM) ${ }^{(8)}$. Los exámenes de imagen son de vital importancia en el estudio de GPA, y constituyen un pilar importante en los criterios diagnósticos. Un resumen de sus indicaciones se muestra en la Tabla 1.

La TC es la técnica de elección para la caracterización de las manifestaciones en GPA, por su alta resolución espacial, amplia disponibilidad y menor costo. Presenta alto rendimiento diagnóstico en patología de cavidades paranasales, y es el examen con mejor relación costo-efectividad en la evaluación del tórax, especialmente del pulmón, árbol traqueobronquial, mediastino y estructuras vasculares.

La RM es de mayor utilidad en las manifestaciones cardiacas, encefálicas y oculares que, aunque inusuales en GPA, exigen un diagnóstico precoz y una caracterización precisa ${ }^{(8,9)}$.

Hay que considerar que los pequeños vasos afectados por el proceso de vasculitis no van a ser visibles con esta ni ninguna otra técnica de imagen tomográfica, ya que por su pequeño tamaño exceden la resolución espacial de la TC y RM. Lo que suelen detectar estos exámenes son los fenómenos inflamatorios o necróticos asociados, que logren constituir una lesión macroscópica en un órgano o tejido.

EI PET/CT tampoco es capaz de detectar la vasculitis de pequeño vaso per se, aunque al ser un estudio funcional, puede pesquisar la inflamación en un tejido macroscópicamente normal, siempre que sea de un tamaño suficiente para ser detectado. Por este motivo el PET/CT puede contribuir a un diagnóstico precoz, con positividad en casos con TC normal, y hallazgos que incluso preceden a las alteraciones de laboratorio(10). Además, puede diferenciar tejido inflamatorio granulomatoso, que suele presentar alta captación de glucosa marcada, del tejido necrótico o fibrótico, que no capta F18-FDG. Por este motivo, el $\mathrm{PET} / \mathrm{CT}$ presenta utilidad en el control del tratamiento y seguimiento, ya que permite evaluar la actividad de la enfermedad, y detectar precozmente recaídas ${ }^{(11)}$, Varios estudios han demostrado una correlación entre



Figura 1: a) Área de necrosis geográfica (flechas). b) proceso inflamatorio mixto (crónico y agudo) con microabsceso en el centro (flechas largas) y dos células gigantes multinucleadas en el interior de un granuloma de bordes mal definidos (flechas cortas). c) vasculitis arteriolar con infiltración de la íntima por linfocitos, células plasmáticas e histiocitos (flechas). 
Tabla 1. Frecuencia de compromiso clínico-imagenológico y técnica diagnóstica de elección.

\begin{tabular}{|c|c|c|}
\hline Laboratorio & Frecuencia & Técnica diagnóstica \\
\hline $\begin{aligned}-\mathrm{ANCA}+ & \\
& \text { ANCA PR3 } \\
& \text { ANCA MPO }\end{aligned}$ & $\begin{array}{l}90 \% \\
80 \% \\
10 \%\end{array}$ & ELISA \\
\hline Síntomas constitucionales & $30-80 \%$ & Examen clínico \\
\hline $\begin{array}{l}\text { Vía aérea superior } \\
\text { - Cavidades paranasales } \\
\text { - Cavidad nasal } \\
\text { - Oído medio/mastoides } \\
\text { - Engrosamiento subglótico }\end{array}$ & $\begin{array}{l}100 \% \\
100 \% \\
30-50 \% \\
30-50 \%\end{array}$ & $\begin{array}{l}\text { TC } \\
\text { TC } \\
\text { TC } \\
\text { TC }\end{array}$ \\
\hline $\begin{array}{l}\text { Vía aérea inferior } \\
\text { - Masa/nódulo pulmonar } \\
\text { - Nódulo pulmonar cavitado } \\
\text { - Condensación/vidrio esmerilado } \\
\text { - Engrosamiento tráquea } \\
\text { - Bronquiectasias } \\
\text { - Derrame pleural } \\
\text { - Hemorragia alveolar difusa }\end{array}$ & $\begin{array}{l}70-90 \% \\
50 \% \\
25 \% \\
16-50 \% \\
10-20 \% \\
25 \% \\
10 \%\end{array}$ & $\begin{array}{l}\text { TC ó PET/CT } \\
\text { TC ó PET/CT } \\
\text { TC } \\
\text { TC } \\
\text { TC } \\
\text { TC } \\
\text { TC }\end{array}$ \\
\hline $\begin{array}{l}\text { Renal } \\
\qquad \text { Glomerulonefritis necrotizante }\end{array}$ & $40-100 \%$ & Examen de Orina \\
\hline $\begin{array}{l}\text { Otros órganos } \\
\text { - Articular } \\
\text { - Piel y mucosas } \\
\text { - Ojo, nervio óptico } \\
\text { - Sistema nervioso central y periférico } \\
\text { - Pericardio } \\
\text { - Miocardio } \\
\text { - Gastrointestinal } \\
\text { - Compromiso GV (Aorta y sus ramas) } \\
\text { - Urogenital }\end{array}$ & $\begin{array}{l}50-70 \% \\
14-50 \% \\
30-60 \% \\
10-54 \% \\
3-15 \% \\
\text { Raro } \\
4-24 \% \\
\text { Raro } \\
\text { Raro }\end{array}$ & $\begin{array}{l}\text { RM ó Ecografía partes blandas } \\
\text { Examen clínico } \\
\text { RM } \\
\text { RM } \\
\text { TC ó PET/CT } \\
\text { RM ó PET/CT } \\
\text { TC } \\
\text { AngioTC, PET/CT } \\
\text { UroTC ó RM }\end{array}$ \\
\hline
\end{tabular}

la intensidad de captación de F18-FDG y la actividad de la enfermedad medida con índices clínicos como el score de Birmingham ${ }^{(11)}$.

El rol de la ecografía se reserva principalmente para la evaluación del compromiso vascular y torácico extra pulmonar. En particular, el ultrasonido es capaz de detectar y caracterizar el compromiso cardiaco, evaluando masas atriales y engrosamientos septales o de la pared aórtica, raros pero posibles de encontrar en GPA, siendo también útil en objetivar la regresión de las anomalías ${ }^{(9)}$.

Existe consenso en que la radiografía simple no debe ser usada para la evaluación de las manifestaciones de las vasculitis sistémicas, ya que no aportan en valorar su extensión ni evolutividad ${ }^{(12)}$.

\section{Manifestaciones Usuales Tracto respiratorio alto}

El compromiso sinusal es uno de los más frecuentes, y es fácilmente detectable con TC. Los hallazgos característicos son el engrosamiento mucoso pansinusal y la existencia de nódulos en partes blandas que realzan con el medio de contraste ${ }^{(8)}$. En algunos casos se pueden ver alteraciones osteo-cartilaginosas, la más frecuente perforación del tabique nasal, lámina papirácea, y paredes de cavidad nasal. La RM es menos sensible que la TC en detectar destrucción ósea ${ }^{(13)}$. EI PET/CT también es altamente sensible en compromiso sinusal, con alta captación mucosa, cartilaginosa y ósea en etapas precoces, aún con TC normal ${ }^{(14)}$ (Figura 2). 


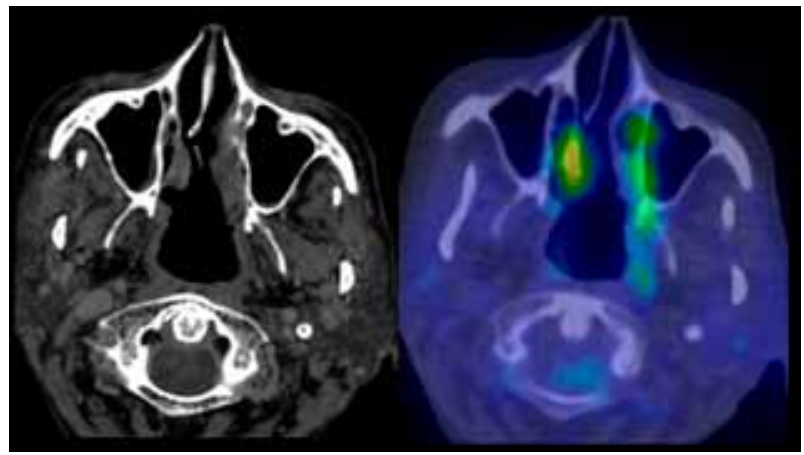

Figura 2: Corte axial de cavidades paranasales muestra $P E T / C T$ (derecha) con marcada hipercaptación de F18-FDG en estructuras óseas de seno maxilary pterigoides izquierdo, y cornete inferior derecho, con TC normal (izquierda).

La otitis y mastoiditis también son frecuentes, visibles en imágenes como ocupación del oído medio y celdas mastoideas por material con densidad de partes blandas.

La estenosis subglótica es una manifestación frecuente, en hasta $50 \%$ de los pacientes ${ }^{(15)}$, la mayoría de las veces asintomática, haciéndose sintomática en casos avanzados con estenosis de más del $80 \%$ del lumen ${ }^{(15)}$. Puede presentarse al momento del diagnóstico o tardíamente en pacientes con GPA en tratamiento, como un signo precoz de recaída ${ }^{(16)}$.

La afectación traqueal ocurre en el $16-50 \%$ de los casos, típicamente en pacientes con enfermedad multisistémica ${ }^{(8,13)}$. El compromiso circunferencial incluyendo la membrana posterior de la tráquea es su característica distintiva, permitiendo el diagnóstico diferencial con la policondritis recidivante y traqueobroncopatía osteocondroplástica, que sólo afectan la porción cartilaginosa, respetando la membranosa ${ }^{(8)}$. En una serie de 51 pacientes con GPA estudiados con TC, Daum et al. ${ }^{(17)}$ reportan traqueobronquitis ulcerativa en $60 \%$, estenosis subglótica en $17 \%$, y estenosis traqueal o bronquial en $13 \%$.

EI PET/CT muestra alta sensibilidad en la detección de compromiso torácico en GPA activa, mayor al observado en otras vasculitis ANCA positivas, con sensibilidad de $100 \%$ en compromiso del tracto respiratorio y vascular torácico, aunque con baja sensibilidad en lesiones oculares, de piel o sistema nervioso central/periférico ${ }^{(11)}$. La RM suele no agregar información extra a la aportada por TC y PET/CT en el tracto respiratorio alto ${ }^{(11)}$.

\section{Pulmón}

El compromiso pulmonar en GPA ha sido extensamente descrito mediante TC, y este examen suele ser suficiente para demostrarlo y caracterizarlo. Los nódulos y masas pulmonares son el hallazgo radiológico más frecuente en GPA, presente en $70-90 \%$ de los pacientes ${ }^{(8,18)}$. Pueden presentar distribución periférica o peribroncovascular, generalmente son bilaterales, y tienden a cavitar especialmente aquellas lesiones mayores de $2 \mathrm{~cm}^{(18)}$ (Figura 3). Áreas de consolidación y opacidades en vidrio esmerilado pueden verse en $25 \%$ de los casos ${ }^{(18)}$.

Las adenopatías mediastínicas son poco frecuentes como hallazgo aislado, y sólo son visibles en concomitancia con lesiones pulmonares ${ }^{(14)}$. En casos de adenopatías mediastínicas asociadas a engrosamiento traqueal y pulmón normal, debieran plantearse otros diagnósticos como la sarcoidosis, traqueitis infecciosa, linfoma o cáncer broncogénico ${ }^{(8)}$.

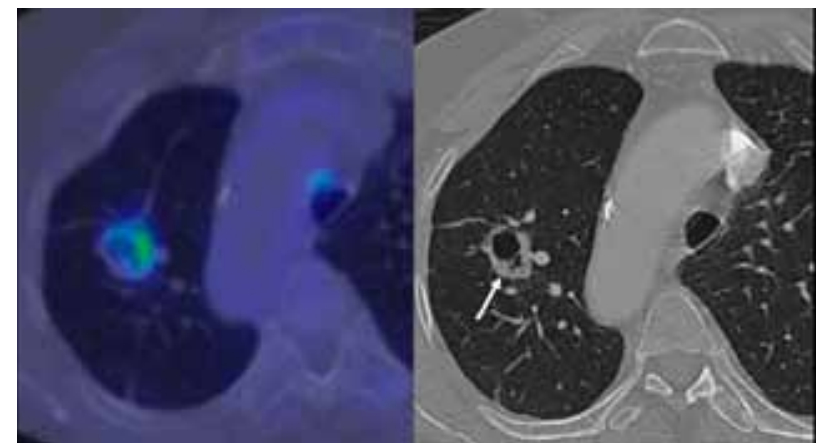

Figura 3: Nódulo pulmonar cavitado en LSI visible en TC (izquierda), con hipercaptación de F18-FDG en el PET/ CT (derecha).

\section{Riñón}

El compromiso renal corresponde a la manifestación clínica más frecuente en nuestro medio ${ }^{(19)}$. Un $80-90 \%$ de los pacientes con GPA presenta evidencia clínica o morfológica de compromiso renal ${ }^{(13,20)}$. Los estudios de imagen tomográficos convencionales (TC, RM) suelen ser negativos en el riñón afectado, salvo cuando se presenta como nódulo o masa renal. EI PET/CT es probablemente el método de imagen más sensible en la detección de compromiso no masiforme, debido a su naturaleza funcional y no puramente anatómica ${ }^{(11)}$, mostrando hipercaptación difusa o parcheada de F18-FDG en la corteza renal en casos de glomerulonefritis acentuada (Figura 4). Recientemente, Fu et al reportaron un caso de detección incidental de compromiso renal bilateral en un paciente con GPA con ecografía y TC normales ${ }^{(21)}$.

\section{Manifestaciones inusuales Órbita}

Si bien no es ampliamente conocido, puede aparecer hasta en un $60 \%$ de los pacientes con GPA e incluso como manifestación inicial ${ }^{(22)}$. Traduce fenómenos isquémicos y necrosis, además de la inflamación granulomatosa. La infiltración inflamatoria orbitaria y periorbitaria con proptosis, generalmente unilateral, es la forma más frecuente de presentación. Menos 


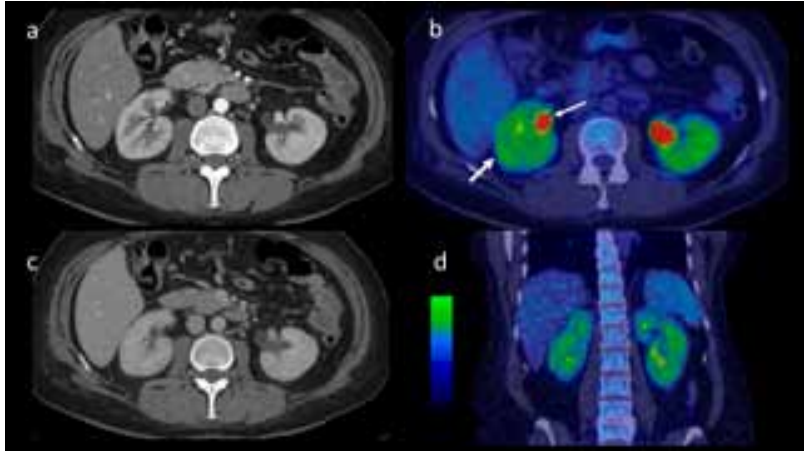

Figura 4: TC en fase arterial (a) y porto-venosa (c) sin alteraciones renales. PET/CT con intensa captación difusa en el parénquima renal (b,d), mayor al hígado y bazo (flecha gruesa). Se muestra pelvis renales con excreción habitual del F18-FDG por la orina (flecha fina).

frecuentemente ocasiona compromiso de la vaina del nervio óptico y fibrosis periocular, hallazgos que pueden ser caracterizados con RM. El compromiso de glándulas lacrimales es infrecuente en GPA y permite el diagnóstico diferencial con sarcoidosis o enfermedades linfoproliferativas ${ }^{(23)}$. La presencia de tejido con densidad de partes blandas bilateral y simétrico ínfero medial en la grasa intraconal, en presencia de una "fosa nasal vacía" es altamente específico de GPA ${ }^{(24,25)}$.

\section{Hueso temporal}

Las manifestaciones óticas se presentan en hasta un $40 \%$ de los pacientes y se producen por extensión directa desde el compromiso nasosinusal, causando una otitis media serosa con o sin afectación mastoidea. Posteriormente la inflamación granulomatosa puede derivar en destrucción ósea, simulando otomastoiditis coalescente, hallazgos evidenciables con $\mathrm{TC}^{(23)}$. También existen casos de otitis que cursan con parálisis facial, que pueden progresar a hipoacusia neurosensorial, exigiendo evaluación ósea de hueso temporal mediante TC, y de los pares craneales afectados mediante $\mathrm{RM}^{(26)}$.

\section{Sistema nervioso central}

Son raras como presentación inicial en GPA, siendo más frecuentes en estadíos avanzados de la enfermedad ${ }^{(27)}$. Lo más frecuente es el compromiso vascular, que puede asociarse a eventos isquémicos (Infarto o accidente isquémico transitorio), hemorragia o trombosis venosa. En RM contrastada, aunque menos frecuente, puede observarse el compromiso hipofisiario caracterizado por aumento de volumen y pérdida de hiperintensidad T1 normal de la hipófisis posterior, engrosamiento y realce del tallo hipofisiario con extensión hipotalámica y compresión secundaria del quiasma óptico ${ }^{(23,28)}$. El compromiso meníngeo (paquimeningitis), con engrosamiento lineal (focal o simétrico) y realce dural, también es infrecuente ${ }^{(23,29,30)}$. En la base de cráneo, también por extensión sinonasal u orbitaria, puede cursar con neuropatías por disfunción de pares craneales principalmente olfatoria y óptica. Ésta última afección debe ser sospechada ante la presencia concomitante de lesiones intracraneales típicas, y es bien caracterizada con RM que mostrará engrosamiento e hiperintensidad T2 del II par ${ }^{(23)}$.

\section{Grandes vasos}

Se han descrito aortitis y periaortitis en GPA, siendo esta última la más frecuente. Se produciría por compromiso de pequeño vaso de la vasa vasorum ${ }^{(31)}$ y/o por extensión del compromiso granulomatoso de la pared arterial hacia los tejidos perivasculares (Figura 5). La aortitis/periaortitis puede ser bien caracterizada con TC contrastada o RM, ambas con excelente precisión por lo que la preferencia de un método por sobre otro dependerá de su disponibilidad además de otros factores como contraindicaciones a los respectivos medios de contraste, presencia de artefactos o consideraciones respecto de la radiación. En ellos se mostrará engrosamiento difuso de la pared arterial con edema y realce tardío(32) (Figura 6). El rol de ambas técnicas en determinar el grado de actividad en vasculitis de grandes vasos permanece aún incierto ${ }^{(33)}$. En una revisión de 23 casos el compromiso aórtico torácico fue de $23 \%$, de arteria subclavia $17 \%$ y carótida interna $17 \%{ }^{(30)}$. Los hallazgos de aneurismas ${ }^{(30)}$ o estenosis vasculares, éstas últimas descritas principalmente en vasculitis de grandes vasos ${ }^{(29)}$, también fueron menos frecuentes. Un análisis retrospectivo de 21 pacientes con GPA estudiados con PET/CT, mostró alta captación de 18F-FDG en la aorta en valores similares a los encontrados en pacientes con vasculitis de grandes vasos, por lo que este examen no permitiría diferenciar entre estas dos entidades ${ }^{(34)}$. Existen publicaciones recientes que asignan un valor agregado a la evaluación ultrasonográfica morfológica y hemodinámica de la vasculitis y la inflamación perivascular, añadiendo factores pronósticos de ateromatosis, importante de detectar y tratar en estos pacientes ${ }^{(35)}$.

La aorta abdominal constituye el segmento afectado con mayor frecuencia (52\%), por sobre el número de casos con afectación del segmento torácico o subclavio ${ }^{(30)}$. En éstos pacientes, que además cursan con dolor abdominal, la GPA también puede ocasionar fibrosis retroperitoneal concomitante a la periaortitis ${ }^{(31)}$.

\section{Corazón}

Ocurre entre un $3,3 \%$ y un $15 \%$ de los pacientes con $\mathrm{GPA}^{(13,36)}$, y aunque potencialmente fatal si no se trata oportunamente, no se asocia a peor pronósti$\mathrm{co}^{(36)}$. Es frecuentemente subdiagnosticado por ser a menudo de presentación subclínica ${ }^{(37)}$. Diversos 


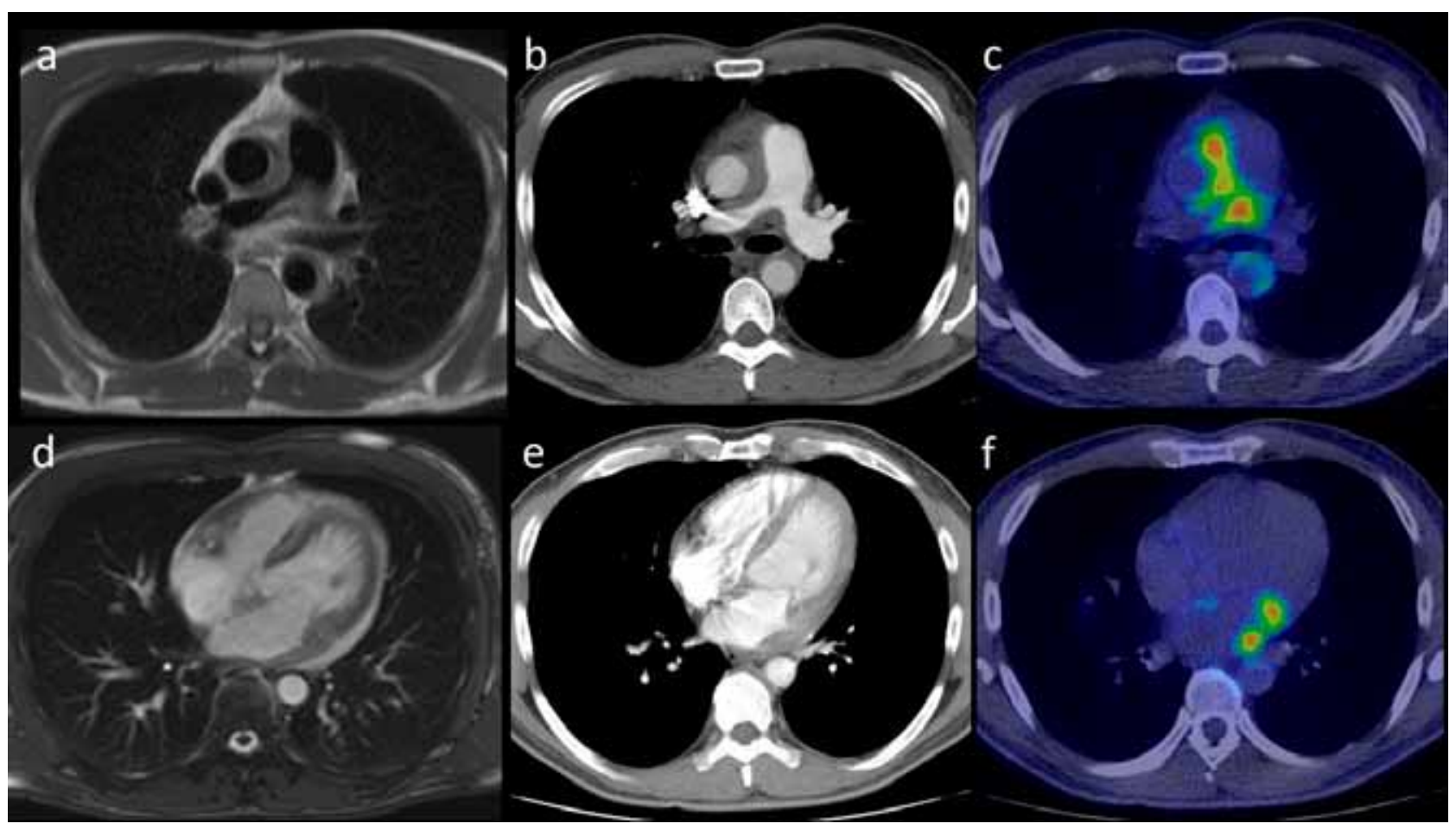

Figura 5: a) Secuencia HASTE axial que muestra engrosamiento periaórtico y alrededor de la arteria pulmonar derecha, lo que determina disminución de su calibre. Mismo corte en TC contrastada (b) y PET/CT (c). d) Secuencia SSFP sangre blanca corte axial que muestra tejido sólido que compromete ambos surcos auriculoventriculares rodeando las arterias coronarias. PET/CT con focos hipercaptantes en estas mismas localizaciones (f). Las lesiones no son evidentes en la TC contrastada (e).



Figura 6: TC contrastada con engrosamiento periaórtico (a), que al PET/CT presenta intensa hipercaptación irregular de F18-FDG (b). c) Secuencia HASTE corte axial con engrosamiento periaórtico a nivel del cayado. d) Secuencia T1 con saturación grasa corte axial que demuestra impregnación tardía del tejido sólido periaórtico. 
estudios sugieren que una aproximación inicial con ecocardiografía transtorácica seguida de evaluación con RM cardiaca contrastada son las herramientas más sensibles para detectar anomalías en pacientes con GPA, y parecen ser también métodos confiables para seguimiento y monitoreo de la terapia en estos pacientes ${ }^{(37,38)}$. Un estudio francés analizó retrospectivamente el compromiso cardiaco en 31 pacientes con GPA confirmada ${ }^{(37)}$. Concluyeron que un $61 \%$ de los pacientes presentó al menos una anomalía cardiaca atribuible a GPA, que ésta se correlacionaba además con el tiempo transcurrido desde el diagnóstico (mayor de 18 meses) y que los pacientes clínicamente sintomáticos tenían lesiones más extensas. Entre los hallazgos principales en RM destacan la miocarditis granulomatosa (con hipoquinesia ventricular y realce tardío subepicárdico con gadolinio), disfunción ventricular izquierda $(\mathrm{FE}<45)$ y pericarditis ${ }^{(37)}$. Otras alteraciones descritas incluyen arteritis de las coronarias y compromiso valvular mitral incidental ${ }^{(9)}$. El PET-CT también ha demostrado alta positividad en lesiones cardiacas con excelente correlación con las alteraciones evidentes en la RM. Su utilidad en el control post tratamiento también ha sido sugerido en algunas series ${ }^{(11)}$.

\section{Tracto Gastrointestinal}

Si bien el compromiso a este nivel resulta desconocido, debe considerarse ya que puede afectar tanto al intestino delgado como al colon, con complicaciones serias que pueden requerir manejo quirúrgico urgente. Se describe que afecta a entre un $10 \%$ y $24 \%$ de los pacientes durante los dos primeros años del diagnóstico, aunque la confirmación histológica se logra en pocos casos, mayormente en autopsias. Debe sospecharse ante cuadros que sugieran una enfermedad inflamatoria intestinal, con síntomas como dolor abdominal y sangrado gastrointestinal. Estos síntomas traducen los hallazgos macroscópicos detectados por endoscopía, como ulceraciones, necrosis parietal intestinal y perforación ${ }^{(39)}$.

\section{Otros sistemas}

También está descrito el compromiso en glándulas salivales, lesiones cutáneas y algunas manifestaciones articulares, frecuentes durante episodios de enfermedad activa ${ }^{(11)}$. EL PET/CT puede ayudar a detectar algunas de estas alteraciones, a nivel $\operatorname{articular}^{(14)} \mathrm{O}$ en glándulas salivales ${ }^{(11)}$.

\section{Tratamiento}

El tratamiento consta de dos fases: una primera fase de inducción, de 3 a 6 meses, cuyo objetivo es lograr la remisión, y una segunda fase de mantención, de 12-24 meses, para consolidar la remisión y evitar recaídas $^{(2)}$. Para la inducción en la forma sistémica de la enfermedad se recomiendan corticoides en altas dosis asociado a otro inmunosupresor, ciclofosfamida o rituximab; algunos casos pueden requerir plasmaféresis. Para formas localizadas sin compromiso de órganos vitales está recomendado el uso de corticoides asociados a metotrexato o micofenolato mofetil. En la fase de mantención, se recomienda la combinación de bajas dosis de corticoides orales con azatioprina o metotrexato y, en algunos casos, se puede considerar micofenolato mofetil y leflunomida. El uso de rituximab también ha demostrado mantener remisión de la enfermedad con menor porcentaje de recaídas. Se recomienda el uso sistemático de cotrimoxazol para prevenir recaídas e infecciones por Pneumocystis jirovecii ${ }^{(2,3,40)}$.

Los autores declaran no tener conflictos de interés.

\section{Referencias}

1. Jeannette JC, Falk RL, Bacon PA, Basu N, Cid MC, Ferrario F, et al. 2012 Revised International Chapel Hill Consensus Conference Nomenclature of Vasculitides. Arthritis Rheum 2013; 65: 1-11.

2. Comarmond C, Cacoub P. Granulomatosis with polyangiitis (Wegener): Clinical aspects and Conference Autoimmun Rev 2014; 13: 1121-1125.

3. Shi L. Anti-neutrophil cytoplasmic antibody-associated vascutilis: Prevalence, treatment, and outcomes. Rheumatol Int 2017; 37: 1779-1788.

4. Lutalo P, Cruz D. Diagnosis and classification of granulomatosis with poliangiitis (aka Wegener's granulomatosis). J Autoimmun 2014; Volumen 48-49: 94-98.

5. Unizony S, Villarreal M, Miloslavsky E, Lu N, Merkel P, Spiera R, et al. Clinical outcomes of treament of antineutrophil cytoplasmic antibody (ANCA)-associated vasculitis based on ANCA type. Ann Rheum Dis 2016; 75: 1166-1169.

6. Mark EJ, Flieder DB, Matsubara O. Treated Wegener's granulomatosis: Distinctive pathological findings in the lungs of 20 patients and what they tell us about the natural history of the disease. Hum Pathol 1997; 28 : 450-458.

7. Yi ES, Colby TV. Wegener's granulomatosis. Semin Diagn Pathol 2001; 18: 34-46.

8. Martinez F, Chung JH, Digumarthy SR, Kanne JP, Abbott GF, M, Jo-Anne O, Shepard J-A O, et al. Manifestations of Wegener Granulomatosis at Chest CT: Radiologic- Pathologic Correlation. RadioGraphics 2012; 32: 51-69.

9. Gał ska R, Kulawiak-Gał ska D, Czuszy ska Z, Masiak A, Zdrojewski Z, Gruchała M. A rare case of complex cardiac involvement in granulomatosis with polyangiitis. Pol Arch Intern Med 2017; 127: 63-65.

10. De Geeter F, Gykiere P. F-FDG PET imaging of granuIomatosis with polyangiitis -Wegener's Syndrome. Hell J Nucl Med 2016; 19: 53-56.

11. Soussan M, Abisror N, Abad S, Nunes H, Terrier B, Pop G, et al. FDG-PET/CT in patients with ANCAassociated vasculitis: Case-series and literature review. Autoimmunity Reviews 2014;13: 125-131.

12. Castañer E, Alguersuari A, Gallardo X, Andreu M, Pallardó Y, Mata JM, et al. When to suspect pulmonary 
vasculitis: Radiologic and clinical clues. Radiographics 2010; 30: 33-53.

13. Lynch JP, Tazelaar H. Wegener Granulomatosis (Granulomatosis with Polyangiitis): Evolving Concepts in Treatment. Semin Respir Crit Care Med 2011; 32: 274.297.

14. Nelson DR, Johnson GB, Cartin-Ceba R, Specks U. Characterization of F-18 Fluorodeoxyglucose PET/CT in Granulomatosis with Polyangiitis. Sarcoidosis Vasc Diffuse Lung Dis 2016; 32: 342-52.

15. Massa M, Emery NC, Bosio M, Finn BC, Bruetman JE, Young P. Estenosis subglótica y granulomatosis con poliangeítis (Wegener) en dos casos. Rev Med Chile 2014; 142: 382-385.

16. Langford CA, Sneller MC, Hallahan CW, Hoffman GS, Kammerer WA, Talar Williams C, et al. Clinical features and therapeutic management of subglottic stenosis in patients with Wegener's granulomatosis. Arthritis Rheum 1996; 39: 1754-1760.

17. Daum TE, Specks U, Colby TV, Edell ES, Brutinel MW, Prakash UB, et al. Tracheobronchial involvement in Wegener's granulomatosis. Am J Respir Crit Care Med 1995; 151: 522-526.

18. Airways. En: Naidich DP, Webb WR, Muller NL, Vlahos I, Krinsky GA, Ed. Computed Tomography and Magnetic Resonance of the Thorax. Philadelphia, PA 19106 USA: Lippincott-Raven Publishers; Third edition 1999: 500-501.

19. Cisternas M, Soto L, Jacobelli S, Marinovic MA, Vargas A, Sobarzo E, et al. Manifestaciones clínicas de la granulomatosis de Wegener y la poliangeítis microscópica en Santiago-Chile, 1990-2001. Rev Med Chile 2005; 133: 273-278.

20. Renaudineau $Y$, Le Meur Y. Renal Involvement in Wegener's Granulomatosis. Clinic Rev Allerg Immunol 2008; 35: 22-29.

21. Fu Z, Liu M, Ziao Li Z, Zhang J, Li Q. Occult Renal Granulomatous Inflammatory Lesions in Granulomatosis with Polyangiitis Detected by 18F-FDG PET/CT. Clin Nucl Med 2017; 42: 707-708.

22. Muller K, Lin JH. Orbital granulomatosis with polyangiitis (Wegener granulomatosis): Clinical and pathologic findings. Arch Pathol Lab Med 2014; 138: 1110-1114.

23. Pakalniskis MG, Berg AD, Policeni BA, Gentry LR, Sato $Y$, Moritani T, et al. The Many Faces of Granulomatosis with Polyangiitis: A Review of the Head and Neck Imaging Manifestations. AJR 2015; 205: W619-W629.

24. Muller K, Lin JH. Orbital granulomatosis with polyangiitis (Wegener granulomatosis): clinical and pathologic findings. Arch Pathol Lab Med 2014; 138: 1110-1114.

25. Perry, S, Rootman J, White VA. The Clinical and Pathologic Constellation of Wegener Granulomatosis of the Orbit. Ophthalmology 1997; 104: 683-694.

26. Wang JC, Leader BA, Crane RA, Koch BL, Smith MM, Ishman SL. Granulomatosis with polyangiitis presenting as facial nerve palsy in a teenager. Int $J$ Pediatr Otorhinolaryngol 2018; 107: 160-163.

27. Izquierdo Velásquez JC, Romero Moreno LF. Internal
Auditory Canal Dural Enhancement in Granulomatosis with Polyangeitis Disease. Case Rep Neurol Med 2018; Volumen 2018: Article ID 2171434, 3 pages

28. De Parisot A, Puéchal X, Langrand C, Raverot G, Gil H, Perard $\mathrm{L}$, et al. Pituitary involvement in granulomatosis with polyangiitis: report of 9 patients and review of the literature. Medicine 2015; 94: e748.

29. Kaga H, Komatsuda A, Saito M, Nara M, Omokawa A, Togashi M, et al. Anti-neutrophil Cytoplasmic Antibodyassociated Vasculitis Complicated by Periaortitis and Cranial Hypertrophic Pachymeningitis: A Report of an Autopsy Case. Intern Med 2018; 57: 107-113.

30. Ozaki T, Maeshima K, Kiyonaga Y, Torigoe M, Imada $\mathrm{C}$, Hamasaki $\mathrm{H}$, et al. Large-vessel involvement in granulomatosis with polyangiitis successfully treated with rituximab: A case report and literature review. Mod Rheumatol 2017; 27: 699-704.

31. González Revilla EM, Fernandez AA, Ramirez MT, Pardo SC, Moragues MA. Retroperitoneal fibrosis with periaortitis: A case report of an unusual form of presentation of granulomatosis with polyangiitis. Respir Med Case Rep 2016; 19: 121-124.

32. Baliyan V, Verdini D, Meyersohn NM. Noninvasive aortic imaging. Cardiovasc Diagn Ther. 2018; 8(Suppl 1): S3-S18.

33. Barra L, Kanji T, Malette J, Pagnoux C. Imaging modalities for the diagnosis and disease activity assessment of Takayasu's arteritis: A systematic review and metaanalysis. Autoimmun Rev. 2018; 17: 175-187.

34. Kemna MJ, Bucerius J, Drent M, Vöö S, Veenman M, Van Paassen $\mathrm{P}$, et al. Aortic 18F-FDG uptake in patients suffering from granulomatosis with polyangiitis. Eur $\mathrm{J}$ Nucl Med Mol Imaging 2015; 42: 1423-1429.

35. Pacholczak R, Bazan-Socha S, Iwaniec T, Zar ba L, Kielczewski S, Walocha JA, et al. Endothelial dysfunction in patients with granulomatosis with polyangiitis: A case-control study. Rheumatol Int 2018; 38: 1521-1530.

36. McGeoch L, Carette S, Cuthbertson D, Hoffman GS, Khalidi N, Koening CL, et al. Cardiac Involvement in Granulomatosis with Polyangiitis. J Rheumatol 2015; 42: 1209-1212.

37. Pugnet G, Gouya H, Puéchal X, Terrier B, Kahan A, Legmann $P$, et al. Cardiac involvement in granulomatosis with polyangiitis: A magnetic resonance imaging study of 31 consecutive patients. Rheumatology 2017; 56(6): 947-956.

38. yci ska K, Borowiec A, Zielonka TM, Rusinowicz T, Hadzik-Błaszczyk M, Cieplak M, et al. Echocardiographic Assessment in Patients with Granulomatosis with Polyangiitis. Adv Exp Med Biol 2017; 1022: 27-33.

39. Masiak A, Zdrojewski L, Zdrojewski Z, Bułło-Piontecka B, Rutkowski B. Gastrointestinal tract involvement in granulomatosis with polyangiitis. Prz Gastroenterol 2016; 11: 270-275.

40. Yates M, Watts RA, Bajema IM, Cid MC, Crestani B, Hauser T, et al. EULAR/EDTA recommendations for the management of ANCA-associated vasculitis. Ann Rheum Dis 2016; 75: 1583-1594. 